\title{
The Tale of the Design of Sandwich
}

\section{Yousef Haik*}

Department of Mechanical Engineering, United Arab Emirates University, P O Box 15551, Al Ain, UAE

Assessing the artefacts of the previous century one could see predominantly mechanical constructs, cleverly crafted to perform a task. A pantograph is one such example where the changing of the length ratio provides the required scaling to make a scaled replica of an object. Geneva Mechanisms provide intermittent rotations of various kinds derived from continuous rotations. A stepper motor now provides this function in a better and efficient way. While one sees the diminishing influence of the Geneva Mechanism with sadness from an academic point of view he will not fail to appreciate the enormous potential to provide fractional rotations unleashed by the stepper motor. New technologies are replacing the old ones at a very rapid pace. However the requirement for the function that prompted the original artefact remains. Often additional requirements, which were thought impossible with the old technology, are added to the list and provided by the modern versions of the old artefact. One can witness this effect clearly by comparing a camera from the 60's to a modern digital camera. The question is 'How to develop this new Version' of the old artefact. This is an important question since a major part of the design work to be carried out in the foreseeable future would be developing newer versions of the old artefacts. We believe that the answer lies in adopting a systematic design methodology where the design process is broken into stages and where various design methods can be used to carry out tasks belonging to individual stages. We have identified five basic landmark stages in the design process. They are:

\section{Requirements}

In this stage of the design process customers' statements on requirements are recorded and converted into design needs that can be articulated as intended or targeted design specifications with measurable metrics.

\section{Product Concepts}

In this stage the product functions are established and their intended design specifications from the previous stage are integrated to define the final set of specifications.

\section{Solution Concept}

In this stage the conceptual designs that can meet the specifications are developed and their behaviour and functions are well described. An optimal design is identified according to some well-developed criteria.

\section{Embodiment Design}

This is the stage where physical parts are introduced. A parts tree showing the sub-assemblies and assemblies is developed; Make/buy decisions are made; And a layout of the various parts are identified.

\section{Detail Design}

In this stage the entire details including the material and manufacturing processes are defined first, in such a way that the artefact can be manufactured if needed. The analyses needed to prove the product functionality and safety for the operation are identified and carried out next, in full at this stage. The proven design is then committed for manufacture.

Consider this process on a simple product like a sandwich to understand the potential of the systematic design process.A sandwich is a food item consisting of two pieces of bread with a filling between them. It is said that in 1726 John Montague, the fourth Earl of Sandwich, while spending endless hours playing cards, thought about placing the cheese and the meat between the slices of bread to eat while continue playing without wetting the cards by the food. The simple food item with a humble origin was named after him and became popular ever since. A Franchise provider wants to develop cheese salad sandwiches that are sold in his outlets. These sandwiches are to be made of normal loafs of bread and vegetables and sealed. A systematic design process was employed with the establishment of a 'Design Brief' a document with (i) Product description (ii) product concept (iii) benefits to be delivered (iv) positioning and target price (v) target market (vi) assumptions and constraints (vi) stakeholders (vii) possible features and attributes and (viii) possible areas for innovation as its key elements. Customers were interviewed and their requirements were recorded. Their verbatim included statements like (i) should be sufficient to contain my hunger (ii) should know the contents easily from outside (iii) should know the number of days on the shelf and (iv) I do not like salad dressing. The entire verbatim is then translated into needs for deployment in the design by the design team. The needs were then mapped into measurable characteristics with suitable units of measurement to facilitate drawing the technical specifications. In this process the verbatim 'Should be sufficient to contain my hunger' became 'adequate quantity of bread (need)' and 'weight of bread in grams (characteristic and unit)'. A list of functions was prepared for each item in the sandwich. For example the functions of the bread were identified as (i) keep salad (ii) assure calorie requirement (iii) contain food value nutrients. They were arranged in a hierarchy diagram as purpose functions and action functions forming a function tree. For example the purpose function 'keep Salad' is divided into action functions (i) cover salad (ii) contain salad (iii) support salad and (iv) absorb juices. The purpose functions often help to form the specifications and the specification drawn here was 'keep 20 grams' of salad. Conceptual design for the sandwich decides what constituents such as (i) bread size (ii) salad choice (iii) dressing (iv) spice level and (v) the cheese type that goes to make the sandwich. Carrying out a morphological analysis can identify many options or concepts but a shortlist of 144 were identified from the analysis conducted. This was a unique situation with such large number of viable options. It was therefore decided to have them put on trial sales with 12 options each day for two months in chosen twenty outlets. The data collected provides the basis to choose options. The next step was the embodiment design which starts with the establishment of a parts

*Corresponding author: Yousef Haik, Department of Mechanical Engineering United Arab Emirates University, P O Box 15551, Al Ain, UAE, Tel: 009713 7136576; E-mail: yhaik@uaeu.ac.ae

Received December 16 2013; Accepted December 16, 2013; Published December 23, 2013

Citation: Haik Y (2013) The Tale of the Design of Sandwich. J Appl Mech Eng 2: e125. doi:10.4172/2168-9873.1000e125

Copyright: () 2013 Haik Y. This is an open-access article distributed under the terms of the Creative Commons Attribution License, which permits unrestricted use, distribution, and reproduction in any medium, provided the original author and source are credited. 
tree, bill of quantities with make/buy decisions with due consideration for raw materials and manufacturing. The detail design ensures the complete definition of each piece part from the geometric shape of the bread piece to the label that goes into the making of the sandwich. The detailed design part involves the choice of needed engineering analyses. For a sandwich the geometry of the net and the nesting of the blanks for the package, are the two analyses identified and performed as the needed ones. Having thus completed the design in a systematic way the manufacturing and marketing can be decided based on business considerations. If the outlets are small and space-wise compact, making the sandwich there would be impossible. In these situations derivatives of a platform product like 'Cheese salad sandwich with- - - ' would be more appropriate. On the other hand if there is adequate space and labour is cheap the ingredients can be kept on-site for the customers to choose and sandwiches like 'Tell-us what you choose and we will make them while you wait. This modular design approach can satisfy the customers in a more individual way.

Design remains the fundamental ingredient for all engineering disciplines; we cannot stress enough its value in contributing to the advancement of society. However the most educational system emphasizes the last tail of the design process, detailed design, and ignores the large part where the creativity is involved in the other stages of the design process. The process, is adoptable, even for designing a sandwich, which will expose the solution space with solutions never thought of before. We are hoping that the power of systematic design is understood and more of the design process is introduced in the engineering curriculum. 